Homology, Homotopy and Applications, vol.15(1), 2013, pp.83-100

\title{
CHEVALLEY COHOMOLOGY FOR AERIAL KONTSEVICH GRAPHS
}

\author{
WALID ALOULOU, DIDIER ARNAL AND RIDHA CHATBOURI
}

(communicated by Johannes Huebschmann)

\begin{abstract}
Let $T_{\text {poly }}\left(\mathbb{R}^{d}\right)$ denote the space of skew-symmetric polyvector fields on $\mathbb{R}^{d}$, turned into a graded Lie algebra by means of the Schouten bracket. Our aim is to explore the cohomology of this Lie algebra, with coefficients in the adjoint representation, arising from cochains defined by linear combination of aerial Kontsevich graphs. We prove that this cohomology is localized at the space of graphs without any isolated vertex, any "hand" or any "foot". As an application, we explicitly compute the cohomology of the "ascending graphs" quotient complex.
\end{abstract}

\section{Introduction}

Let $T_{\text {poly }}\left(\mathbb{R}^{d}\right)$ denote the space of skew-symmetric polyvector fields on $\mathbb{R}^{d}$, regraded down by 1 ; thus an ordinary vector field has degree 0 and a homogeneous polyvector field arising as the exterior product of $k \geqslant 1$ ordinary vector fields has degree $k-1$. With this grading, the standard Schouten bracket turns $T_{\text {poly }}\left(\mathbb{R}^{d}\right)$ into a graded Lie algebra. As in $[\mathbf{A G M}]$, we will study the Chevalley cohomology of this graded algebra with coefficients in the adjoint representation. This cohomology is an important tool in the construction of formalities, i.e., quasi isomorphisms between this Lie algebra, and the Lie algebra of polydifferential operators, when these Lie algebras are viewed as algebras up to homotopy (see $[\mathbf{A G M}, \mathbf{K 2}]$ ). See also $[\mathbf{K 1}]$, where the stable (co)homology of various naturally arising subalgebras of $T_{\text {poly }}\left(\mathbb{R}^{d}\right)$ was computed.

As in $[\mathbf{A G M}]$, we restrict attention to cochains $C_{\Delta}$ defined by "aerial" admissible graphs $\Delta$. The graphs under consideration do not have multiple edges but small loops, i.e., loops starting and ending at the same vertex are admitted.

Let $\Delta$ be an "aerial" admissible graph. Given a vertex $A$, we denote by $\operatorname{Star}(A)$ the collection of edges starting at $A$ and by $\operatorname{End}(A)$ that of edges ending at $A$. We will say that $A$ is a hand of $\Delta$ if $\# \operatorname{Star}(A)=1$ and $\# \operatorname{End}(A)=0$ and, likewise, we will say that $A$ is a foot of $\Delta$ if $\# \operatorname{Star}(A)=0$ and $\# \operatorname{End}(A)=1$.

This work was supported by the CMCU contract (Hubert Curien Utique) 09/G 1502. W. Aloulou and R. Chatbouri thank the Université de Bourgogne and D. Arnal the Faculté des Sciences de Monastir for their kind hospitalities during their stays.

Received November 3, 2010, revised June 7, 2011, March 30, 2012; published on March 11, 2013. 2000 Mathematics Subject Classification: 17B56, 53D50, 05C90.

Key words and phrases: Kontsevich graphs, Chevalley cohomology.

Article available at http://intlpress.com/HHA/v15/n1/a5 and doi:10.4310/HHA.2013.v15.n1.a5

Copyright (C) 2013, International Press. Permission to copy for private use granted. 
The collection $C_{\Delta}$ of cochains constitutes a subcomplex; in particular, it is closed under the coboundary operator. Furthermore, the coboundary operator corresponds to a series of blow-ups for vertices of $\Delta$ followed by the addition of an edge. The resulting cohomology vanishes in small degrees (see, for instance, $[\mathbf{A G M}]$, where it is proved that the cohomology is zero in degrees $\leqslant 3$ ).

In $[\mathbf{A A C 1}]$ and $[\mathbf{A A C 2}]$ we considered only small subalgebras of $T_{\text {poly }}\left(\mathbb{R}^{d}\right)$, where "small" means that we restricted attention to particular classes of graphs $\Delta$.

Here we explore $T_{\text {poly }}\left(\mathbb{R}^{d}\right)$ itself (i.e., we consider all the admissible graphs $\Delta$ ).

We first note that each connected component of an admissible graph cannot be trivial, i.e., such a connected component is neither the graph with a single vertex and no edge nor the graph with two vertices and one edge.

Then, using an explicit homotopy, we localize the cohomology at the space of cochains defined by linear combinations of graphs without any hand or foot.

In the last section, we study a certain quotient complex of graphs, more precisely, the space of ascending graphs on which another homotopy can be defined. We then compute the corresponding cohomology completely. As in [AAC1, AAC2], the cohomology is freely generated by odd wheels products.

We thank the referee for his valuable suggestions, which led to a better result in Proposition 6.3 and to better proofs in Section 7.

\section{Definitions and notations}

Let us first recall the Schouten bracket of two polyvector fields in $T_{\text {poly }}\left(\mathbb{R}^{d}\right)$. Consider the polyvector $\alpha=\sum_{i_{1} \cdots i_{k}} \alpha^{i_{1} \cdots i_{k}} \partial_{i_{1}} \wedge \cdots \wedge \partial_{i_{k}}$, with degree $\operatorname{deg}(\alpha)=k-1$, and $\beta=\sum_{j_{1} \cdots j_{\ell}} \beta^{j_{1} \cdots j_{\ell}} \partial_{j_{1}} \wedge \cdots \wedge \partial_{j_{\ell}}$, with degree $\ell-1$.

As in $[\mathbf{A A C 1}]$, we put

$$
\nabla_{\alpha} \beta=\sum_{r=1}^{k}(-1)^{r-1} \sum_{\substack{i_{1}, \ldots, i_{k} \\ j_{1}, \ldots, j_{\ell}}} \alpha^{i_{1} \cdots i_{k}}\left(\partial_{i_{r}} \beta^{j_{1} \cdots j_{\ell}}\right) \partial_{i_{1}} \wedge \cdots \widehat{\partial_{i_{r}}} \cdots \wedge \partial_{i_{k}} \wedge \partial_{j_{1}} \wedge \cdots \wedge \partial_{j_{\ell}}
$$

Then the Schouten bracket of $\alpha$ and $\beta$ is

$$
[\alpha, \beta]_{S}=(-1)^{\operatorname{deg}(\alpha)} \nabla_{\alpha} \beta-(-1)^{(\operatorname{deg}(\alpha)+1) \operatorname{deg}(\beta)} \nabla_{\beta} \alpha .
$$

Let $C^{\prime}: \bigwedge^{n} T_{\text {poly }}\left(\mathbb{R}^{d}\right) \longrightarrow T_{\text {poly }}\left(\mathbb{R}^{d}\right)$ be a skew-symmetric (for deg) mapping with degree $\operatorname{deg}\left(C^{\prime}\right)$. If $\partial^{\prime}$ is the usual Chevalley coboundary operator, then $\partial^{\prime} C^{\prime}$ is, by definition,

$$
\begin{aligned}
\left(\partial^{\prime} C^{\prime}\right)\left(\alpha_{0}, \ldots, \alpha_{n}\right) & \\
= & \sum_{i=0}^{n}(-1)^{i} \varepsilon_{\operatorname{deg}(\alpha)}(i, 0 \ldots \widehat{i} \ldots n)(-1)^{\operatorname{deg}\left(C^{\prime}\right) \operatorname{deg}\left(\alpha_{i}\right)}\left[\alpha_{i}, C^{\prime}\left(\alpha_{0}, \ldots \widehat{\alpha_{i}} \ldots, \alpha_{n}\right)\right]_{S} \\
& -\sum_{i<j} \varepsilon_{\operatorname{deg}(\alpha)}(i, j, 0, \ldots \widehat{i} \ldots \widehat{j} \ldots n)(-1)^{i+j-1} C^{\prime}\left(\left[\alpha_{i}, \alpha_{j}\right]_{S}, \alpha_{0}, \ldots \widehat{\alpha_{i}} \ldots \widehat{\alpha_{j}} \ldots, \alpha_{n}\right),
\end{aligned}
$$

where the $\operatorname{sign} \varepsilon_{\operatorname{deg}(\alpha)}(\sigma)$ is the sign of the permutation $\sigma$ acting on $\left(\alpha_{0}, \ldots, \alpha_{n}\right)$ in the graded sense. 
However, if we change the graduation by putting $|\alpha|=\operatorname{deg}(\alpha)+1$, then each $n$-linear skew-symmetric mapping becomes a $n$-linear symmetric mapping. With this shift of degree, $T_{\text {poly }}\left(\mathbb{R}^{d}\right)$ becomes a Lie $\{1\}$ algebra, and the Chevalley cohomology operator becomes $\partial$, acting on symmetrized mappings as follows (see [AAC1]):

Proposition 2.1 (Symmetrized cohomology). If $C$ is a n-linear, | |-symmetric mapping, then its coboundary $\partial C$ is

$$
\begin{aligned}
(\partial C)\left(\alpha_{0}, \ldots, \alpha_{n}\right)= & \sum_{i=0}^{n}\left(\varepsilon_{|\alpha|}(i, 0 \ldots \widehat{i} \ldots n)(-1)^{|C|\left(\left|\alpha_{i}\right|-1\right)} \nabla_{\alpha_{i}} C\left(\alpha_{0}, \ldots \widehat{\alpha_{i}} \ldots, \alpha_{n}\right)\right. \\
& \left.+(-1)^{|C|} \varepsilon_{|\alpha|}(0 \ldots \widehat{i} \ldots n, i) \nabla_{C\left(\alpha_{0}, \ldots \widehat{\alpha_{i}} \ldots, \alpha_{n}\right)} \alpha_{i}\right) \\
& -\sum_{i \neq j} \varepsilon_{|\alpha|}(i, j, 0, \ldots \widehat{i} \ldots \widehat{j} \ldots n) C\left(\nabla_{\alpha_{i}} \alpha_{j}, \alpha_{0}, \ldots \widehat{\alpha_{i}} \ldots \widehat{\alpha_{j}} \ldots, \alpha_{n}\right) .
\end{aligned}
$$

From now on, we study this operator $\partial$. As usual, we denote by $Z^{n}$ the space of $n$-cocycles and by $B^{n}$ the space of $n$-coboundaries. The $n^{\text {th }}$ cohomology space is the quotient $H^{n}(\partial)=Z^{n} / B^{n}$.

\section{Aerial graphs and cochains}

In this article, we are only looking for cochains defined by the use of aerial Kontsevich graphs as in $[\mathbf{A G M}]$.

An aerial Kontsevich graph $\Delta$ is a graph with vertices and edges. We label the vertices by capital letters $A, \ldots, Z$, the edges $\overrightarrow{M N}$ are oriented: $\overrightarrow{M N}$ is starting from the vertex $M$ and ending at the vertex $N$. For each vertex $M, \operatorname{Star}(M)$ is the collection of edges starting from $M$, and $\operatorname{End}(M)$ is the collection of edges ending at $M$. To define an orientation on the graph $\Delta$, we first put a total ordering $\mathfrak{n}_{M}$ on $\operatorname{Star}(M)$, for each vertex $M$, and denote by $\operatorname{Star}_{\vec{\Delta}}(M)$ the corresponding list of edges starting from $M$. We then equip the set $V=V_{\Delta}$ of vertices of $\Delta$ with a total ordering $\mathfrak{n}(V)$. These orderings define the orientation of $\Delta$, and we denote the oriented graph as

$$
\vec{\Delta}=(\Delta, \mathfrak{n})=\left(\Delta,\left(\mathfrak{n}(V),\left(\mathfrak{n}_{M}\right)_{M \in V}\right)\right) .
$$

It is therefore a list of edges

$$
E(\vec{\Delta})=\left(\operatorname{Star}_{\vec{\Delta}}(A), \ldots, \operatorname{Star}_{\vec{\Delta}}(Z)\right)
$$

if $\left(V, \mathfrak{n}_{V}\right)=\{A<\cdots<Z\}$.

To build the cochain, we add "terrestrial vertices" and "legs" to $\Delta$.

For all $\# V$-tuple $\left(m_{A}, \ldots, m_{Z}\right)$ of natural numbers, we then add $m=m_{A}+\cdots$ $+m_{Z}$ new vertices, denoted $P_{1}^{M}, \ldots, P_{m_{M}}^{M}(M \in V)$ and $m$ new edges (the legs), $L=\left\{\overrightarrow{M P_{k}^{M}}, M \in V, k \leqslant m_{M}\right\}$. We get a new graph $\Delta_{L}$, which is " $\Delta$ with some legs".

We thus put on $\Delta_{L}$ a natural orientation to get $\overrightarrow{\Delta_{L}}$. 
We first extend to $V_{\Delta_{L}}$ the ordering of $V$ by putting

$$
N<P_{k}^{M}, \quad P_{k}^{M}<P_{k^{\prime}}^{M^{\prime}} \text { if } M<M^{\prime} \text { or } M=M^{\prime} \text { and } k<k^{\prime} .
$$

Similarly, we extend the ordering of $\operatorname{Star}_{\Delta}(M)$ to $\operatorname{Star}_{\Delta_{L}}(M)$ by putting

$$
\overrightarrow{M N}<\overrightarrow{M P_{k}^{M}}<\overrightarrow{M P_{k+1}^{M}} .
$$

Now let $\left(x^{1}, \ldots, x^{d}\right)$ denote the coordinates in $\mathbb{R}^{d}$. Suppose $\vec{e}$ is any edge of $\Delta_{L}$ and $t_{\vec{e}}$ any index, $1 \leqslant t_{\vec{e}} \leqslant d$. We write $\partial_{t_{\vec{e}}}$ for $\frac{\partial}{\partial x^{t} \vec{e}}$.

For any vertex $M$ of $\Delta$, if $\operatorname{Star}_{\Delta_{L}}(M)=\left\{\overrightarrow{e_{1}}<\cdots<\overrightarrow{e_{s_{M}}}\right\}$ and $\alpha$ is a $s_{M}$-vector field, then we denote by $\alpha^{t_{\operatorname{star}(M)}}$ its corresponding component

$$
\alpha^{t_{\mathrm{Star}(M)}}=\alpha^{t_{\overrightarrow{e_{1}}} \cdots t_{\overrightarrow{e_{S_{M}}}}}
$$

In the same way, if $\operatorname{End}(M)=\left\{\overrightarrow{e_{1}}, \ldots, \overrightarrow{e_{a_{M}}}\right\}($ or $\operatorname{End}(M)=\emptyset)$ is the set of arrows

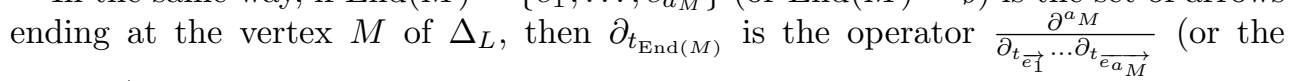
identity).

Finally, if $V_{\Delta}=\left\{M_{1}<\cdots<M_{n}\right\}$ and $\alpha_{1}, \ldots, \alpha_{n}$ are $n$ polyvector fields, then we put, as in $[\mathbf{K} \mathbf{1}], B_{\overrightarrow{\Delta_{L}}}\left(\alpha_{1}, \ldots, \alpha_{n}\right)=0$ except if, for every $i, \alpha_{i}$ is a $s_{M_{i}}$-polyvector field. In this case we put

$$
B_{\overrightarrow{\Delta_{L}}}\left(\alpha_{1}, \ldots, \alpha_{n}\right)=\sum_{\vec{e}, t \vec{e}=1}^{d} \prod_{i=1}^{n} \partial_{t_{\operatorname{End}\left(M_{i}\right)}} \alpha_{i}^{t_{\operatorname{Star}\left(M_{i}\right)}} \partial_{t_{\operatorname{End}\left(P_{1}^{M_{1}}\right)}} \wedge \cdots \wedge \partial_{t_{\operatorname{End}}\left(P_{m_{M_{n}}}^{M_{n}}\right)} .
$$

We say that $B_{\overrightarrow{\Delta_{L}}}$ is the graph operator associated to $\overrightarrow{\Delta_{L}}$.

Remark 3.1. If we change the ordering inside the lists $\operatorname{Star}(M)$, then the operator $B_{\overrightarrow{\Delta_{L}}}$ becomes $\varepsilon(\sigma) B_{\overrightarrow{\vec{L}_{L}}}$ if $\sigma$ is the permutation of the list $E\left(\overrightarrow{\Delta_{L}}\right)$ defined by the change of ordering. We extend the definition of $B_{\overrightarrow{\Delta_{L}}}$ to any ordering of the list of edges in $\overrightarrow{\Delta_{L}}$ by putting $\sigma\left(\overrightarrow{\Delta_{L}}\right)$ for $\sigma\left(E\left(\overrightarrow{\Delta_{L}}\right)\right)$ and:

$$
B_{\sigma\left(\overrightarrow{\Delta_{L}}\right)}=\varepsilon_{\overrightarrow{\Delta_{L}}}(\sigma) B_{\overrightarrow{\Delta_{L}}}
$$

Thanks to this remark, we can put the legs at the end of the list $\Delta_{L}$, getting the new ordering

$$
\left(\operatorname{Star}_{\vec{\Delta}}\left(M_{1}\right), \ldots, \operatorname{Star}_{\vec{\Delta}}\left(M_{n}\right), \overrightarrow{M_{1} P_{1}^{M_{1}}}, \ldots, \overrightarrow{M_{n} P_{m_{M_{n}}}^{M_{n}}}\right) .
$$

Let us denote by $\varepsilon(L)$ the sign of the corresponding permutation of $E\left(\overrightarrow{\Delta_{L}}\right)$. The cochain $C_{\vec{\Delta}}$ defined by the oriented aerial graph $\vec{\Delta}=(\Delta, \mathfrak{n})$ is, by definition,

$$
C_{\Delta, \mathfrak{n}}\left(\alpha_{1}, \ldots, \alpha_{n}\right)=\sum_{L} \varepsilon(L) B_{\overrightarrow{\Delta_{L}}}\left(\alpha_{1}, \ldots, \alpha_{n}\right) .
$$


We consider here the cochains that are linear combinations of some $C_{\Delta, \mathfrak{n}}$ with constant coefficients

$$
C=C_{\sum_{\Delta, \mathfrak{n}} c_{\Delta, \mathfrak{n}}(\Delta, \mathfrak{n})}=\sum_{\Delta, \mathfrak{n}} c_{\Delta, \mathfrak{n}} C_{\Delta, \mathfrak{n}} .
$$

Due to Remark 3.1, we suppose that, for any $\Delta$, any $M \in V(\Delta)$ and any permutation $\tau_{M}$ of $\operatorname{Star}(M), c_{\Delta, \mathfrak{n}}=\varepsilon\left(\tau_{M}\right) c_{\Delta, \mathfrak{n}^{\tau} M}$, where $\mathfrak{n}^{\tau_{M}}$ is the ordering of edges we get through the action of $\tau_{M}$ on $\mathfrak{n}$.

We consider here only symmetric $C$, i.e., the linear combinations $\delta=\sum c_{\Delta, \mathfrak{n}}(\Delta, \mathfrak{n})$ satisfying, for any $\Delta$, any vertex $M$ and any permutation $\sigma \in \mathfrak{S}\left(V_{\Delta}\right)$, or $\sigma \in \mathfrak{S}(\operatorname{Star}(M)), c_{\Delta, \mathfrak{n}^{\sigma}}=\varepsilon_{\Delta}(\sigma) c_{\Delta, \mathfrak{n}}$, where $\mathfrak{n}^{\sigma}$ is the ordering obtained by the permutation of the vertices by $\sigma$, without modifying the ordering in the $\operatorname{Star}(N)$ (resp. the permutation of edges in each $\operatorname{Star}(M))$, and $\varepsilon_{\Delta}(\sigma)$ the sign of the corresponding permutation of $E(\vec{\Delta})$.

The naturally acting symmetry group is therefore the family of permutations $\rho$ of $E(\vec{\Delta})$, where $\rho=\sigma \circ \prod_{M \in V_{\Delta}} \tau_{M}$, with $\sigma \in \mathfrak{S}\left(V_{\Delta}\right), \tau_{M} \in \mathfrak{S}(\operatorname{Star}(M))$ and the symmetrization of the graph $(\Delta, \mathfrak{n})$ is

$$
\operatorname{Sym}(\Delta, \mathfrak{n})=\sum_{\rho} \varepsilon_{\Delta}(\rho)\left(\Delta, \mathfrak{n}^{\rho}\right)=\sum_{\sigma, \tau_{M}} \varepsilon_{\Delta}(\sigma) \prod_{M \in V_{\Delta}} \varepsilon\left(\tau_{M}\right)\left(\Delta,\left(\mathfrak{n}_{V}^{\sigma},\left(\mathfrak{n}_{M}^{\tau_{M}}\right)\right)\right) .
$$

Example 3.2. The symmetrization of the wheel $W_{3}=(\overrightarrow{A B}, \overrightarrow{B C}, \overrightarrow{C A})$ is thus $\operatorname{Sym}\left(W_{3}\right)=3 W_{3}-3 W_{3}^{\prime}$, where $W_{3}^{\prime}$ is the aerial oriented graph $W_{3}^{\prime}=(\overrightarrow{A C}, \overrightarrow{B A}, \overrightarrow{C B})$. The corresponding operator $C_{\mathrm{Sym}\left(W_{3}\right)}$ is an infinite sum of $B_{W_{3 L}}$ and $B_{W_{3 L}^{\prime}}$.

\section{Coboundary operator for aerial graphs}

Let $(\Delta, \mathfrak{n})$ be an oriented aerial graph; the value of the coboundary operator on $(\Delta, \mathfrak{n})$ is a sum of blow-ups (called proper blow-ups in [AAC2]) of the vertices $P$ of $\Delta$. For $P \in V_{\Delta}$, let us now define this blow-up $\partial_{P}(\Delta, \mathfrak{n})$.

We first add a new vertex $Q$ in $V_{\Delta}$ : we put $\partial_{P Q}\left(V_{\Delta}\right)=V_{\Delta} \sqcup\{Q\}$. With this set of vertices, we then build some new graphs $\partial_{P, Q, I, j} \Delta$ as follows:

If, in $\vec{\Delta}, \operatorname{Star}(P)=\left\{\overrightarrow{e_{1}}<\cdots<\overrightarrow{e_{s_{P}}}\right\}$, then fix a subset $I$ of $\operatorname{End}(P)$ and an index $0 \leqslant j \leqslant s_{P}$.

(a) If $M \neq P$ and $N \neq P$, then we keep the $\Delta$ arrows $\overrightarrow{M N}$ in $\partial_{P, Q, I, j} \Delta$.

(b) The $\Delta$ arrows $\overrightarrow{M P}$ become, in $\partial_{P, Q, I, j} \Delta$, either $\overrightarrow{M P}$ if $\overrightarrow{M P}$ was in $I$ or $\overrightarrow{M Q}$ if it was not the case.

(c) The $\Delta$ arrows $\overrightarrow{P M}=\vec{e}_{k}$ become, in $\partial_{P, Q, I, j} \Delta$, either $\overrightarrow{P M}$ if $k \leqslant j$, or $\overrightarrow{Q M}$ if $k>j$.

(d) Finally, we add the arrow $\overrightarrow{P Q}$ in $\partial_{P, Q, I, j} \Delta$.

Denote by $\operatorname{Star}^{\prime}(M), \operatorname{Star}^{\prime}(P)$ and $\operatorname{Star}^{\prime}(Q)$ the star of the vertices in the new graphs.

For any $t, 1 \leqslant t \leqslant s_{P}+1$, we fix an orientation $\mathfrak{n}_{P, Q}^{t}$ on $\partial_{P, Q, I, j} \Delta$ by keeping the ordering on $V_{\Delta}$ and putting $Q$ somewhere before or after $P$ and the new arrow just 
after $\vec{e}_{t-1}$ in $\operatorname{Star}^{\prime}(P)$. Then, if $p<Q$, we get

$$
\begin{aligned}
& \left(\partial_{P, Q, I, j} \Delta, \mathfrak{n}_{P, Q}^{t}\right) \\
& =\left(\operatorname{Star}^{\prime}(A), \ldots,\left(\vec{e}_{1}, \ldots, \vec{e}_{t-1}, \overrightarrow{P Q}, \ldots, \vec{e}_{j}\right), \ldots,\left(\vec{e}_{j+1}^{\prime}, \ldots, \vec{e}_{s_{P}}^{\prime}\right), \ldots, \operatorname{Star}^{\prime}(Z)\right),
\end{aligned}
$$

and, if $Q<P$,

$\left(\partial_{P, Q, I, j} \Delta, \mathfrak{n}_{P, Q}^{t}\right)$

$=\left(\operatorname{Star}^{\prime}(A), \ldots,\left(\vec{e}_{j+1}^{\prime}, \ldots, \vec{e}_{s_{P}}^{\prime}\right), \ldots,\left(\vec{e}_{1}, \ldots, \vec{e}_{t-1}, \overrightarrow{P Q}, \ldots, \vec{e}_{s_{P}}\right), \ldots, \operatorname{Star}^{\prime}(Z)\right)$.

We multiply $\left(\partial_{P, Q, I, j} \Delta, \mathfrak{n}_{P, Q}^{t}\right)$ by a $\operatorname{sign} \varepsilon_{P, Q, I, j}^{t}$. These signs, which are computed in $[\mathbf{A A C 2}]$, are:

$$
\varepsilon_{P, Q, I, j}^{t}=(-1)^{t-1}(-1)^{\sum_{M<P} s_{M}}(-1)^{\sum_{P<M<Q}\left(s_{P}-j\right) s_{M}} \quad \text { if } P<Q,
$$

respectively,

$$
\varepsilon_{P, Q, I, j}^{t}=(-1)^{t-1}(-1)^{\sum_{M<P} s_{M}}(-1)^{\sum_{Q<M<P} j s_{M}}(-1)^{\left(s_{P}-j\right)(j+1)} \quad \text { if } Q<P .
$$

Definition 4.1 (The blow-up of the vertex $P$ ). We call (proper) blow-up of the vertex $P$ in $(\Delta, \mathfrak{n})$ the sum

$$
\partial_{P}(\Delta, \mathfrak{n})=\sum_{Q} \sum_{\substack{0<i+j<a_{P}+s_{P} \\ \text { or } a_{P}+s_{P}=0}} \sum_{\substack{I \subset \operatorname{End}(P) \\ \# I=i}} \sum_{t=1}^{j+1} \varepsilon_{P, Q, I, j}^{t}\left(\partial_{P, Q, I, j} \Delta, \mathfrak{n}_{P, Q}^{t}\right) .
$$

Example 4.2. Let us consider the following aerial graph, where the numbers $(1), \ldots,(3)$ give the edges ordering:

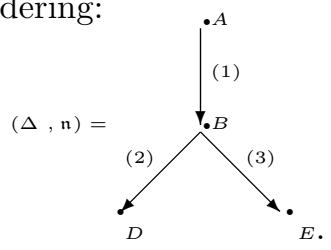

We add the new vertex $C$ and get

$$
\partial_{B}(\Delta, \mathfrak{n})=\partial_{B, C,\{\overrightarrow{A B}\}, 1}(\Delta)+\partial_{B, C,\{\overrightarrow{A C}\}, 1}(\Delta)+\partial_{B, C,\{\overrightarrow{A C}\}, 2}(\Delta) .
$$

Suppose, for instance, we put $C$ between $B$ and $D(A<B<C<D<E)$. We then get the following graphs:

(a) In $\partial_{B, C,\{\overrightarrow{A B}\}, 1}(\Delta)$,
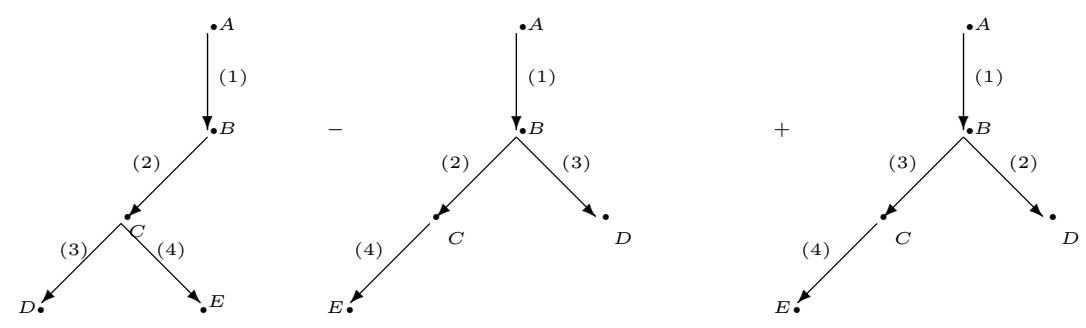
(b) In $\partial_{B, C,\{\overrightarrow{A C}\}, 1}(\Delta)$,

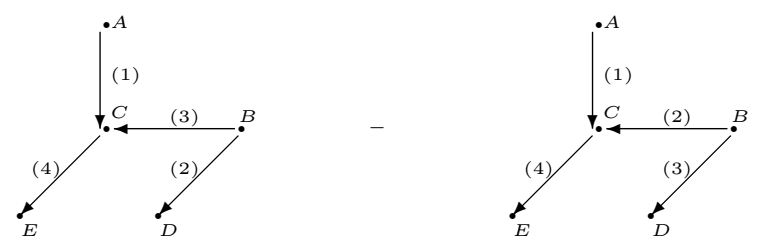

(c) In $\partial_{B, C,\{\overrightarrow{A C}\}, 2}(\Delta)$,
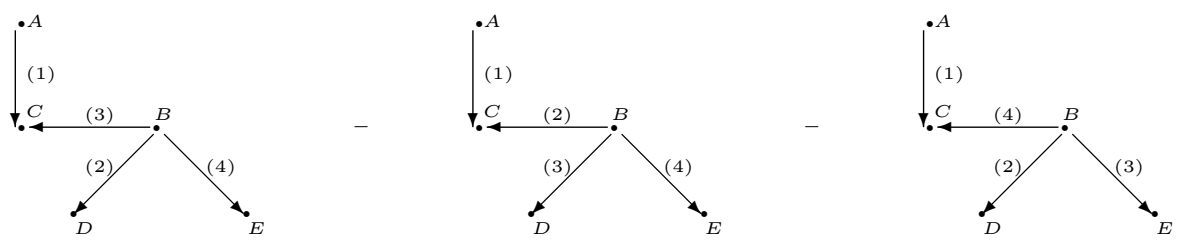

and similarly for each possible position of $C$.

Let us remark that, if $\delta$ is a symmetric combination of graphs $\Delta$, then by construction $\partial_{P} \delta$ is symmetric under permutation of vertices. Moreover, $\partial_{P} \delta$ satisfies $\tau_{M} \partial_{P} \delta=\varepsilon\left(\tau_{M}\right) \partial_{P} \delta$, for any $M \in V_{\partial_{P} \delta}$ and any permutation $\tau_{M}$ of $\operatorname{Star}(M)$. Therefore, if some $\Delta$ appearing in $\delta$ contains the "small loop" $\overrightarrow{P P}$, then some graphs happening in $\partial_{P} \delta$ contain double edges $\overrightarrow{P Q}$, but, by the preceding remark, these graphs disappear in $\partial_{P} \delta$.

As in [AAC2], the following description for the coboundary holds:

Proposition 4.3 (The coboundary $\partial$ on the aerial graphs). Let $\delta=\sum_{\Delta, \mathfrak{n}} c_{\Delta, \mathfrak{n}}(\Delta, \mathfrak{n})$ be a symmetric combination of aerial graphs. Then $\partial C_{\delta}=\sum_{\Delta, \mathfrak{n}} c_{\Delta, \mathfrak{n}} C_{\partial(\Delta, \mathfrak{n})}=C_{\partial \delta}$, where

$$
\partial(\Delta, \mathfrak{n})=-\sum_{P \in V_{\Delta}} \partial_{P}(\Delta, \mathfrak{n})
$$

As usual, we define the cohomology groups on the space $A G$ of linear combinations $\delta$ of aerial graphs. The $n^{\text {th }}$ cohomology group $H^{n}(A G)$ is the quotient $Z^{n} / B^{n}$.

\section{Trivial connected components and degree}

Let us consider a connected component of a graph $\Delta$. If it contains only one vertex (without any edge), or two vertices $A, B$ with only one edge $\overrightarrow{A B}$, then we say it is a trivial component.

Proposition 5.1 (Suppression of trivial components). Up to a coboundary, any cocycle $\delta$ can be written $\delta=\sum c_{\Delta, \mathfrak{n}}(\Delta, \mathfrak{n})$, where each $\Delta$ is a graph without any trivial component. 
Proof. Let us write $\Delta=\Delta_{N} \sqcup \Delta_{T}$, where $\Delta_{T}$ is the union of the trivial components of $\Delta$, and $\Delta_{N}$ is the union of the non-trivial components. Denote by $v_{N}$ the number of vertices in $\Delta_{N}, p_{\Delta}$ the number of "point" components (components with one vertex) and $q_{\Delta}$ the number of "arrows" components (trivial components with two vertices). Remark that, if $q_{\Delta}>1$, then the symmetrization of $\Delta$ vanishes. Thus we can write our cocycle $\delta$ as (omitting to mention the ordering):

$$
\begin{aligned}
\delta & =\sum_{p \geqslant 0} \delta^{(p, 0)}+\delta^{(p, 1)} \\
& =\sum_{p \geqslant 0}\left(\sum_{\Delta, p_{\Delta}=p, q_{\Delta}=0} c_{\Delta}\left(\Delta_{N} \sqcup \Delta_{T}\right)+\sum_{\Delta, p_{\Delta}=p, q_{\Delta}=1} c_{\Delta}\left(\Delta_{N} \sqcup \Delta_{T}\right)\right) .
\end{aligned}
$$

By the definition of $\partial$, we can separate the blow-up of vertices $P$ in $V_{\Delta_{N}}$ with the blow-up of $P$ in $V_{\Delta_{T}}$. Using symmetry, we only look for ordering on $V_{\Delta}$ such that all the vertices in $V_{\Delta_{T}}$ are put down after the vertices in $V_{\Delta_{N}}$. For such a graph $\Delta$,

$$
\partial\left(\Delta_{N} \sqcup \Delta_{T}\right)=\left(\partial \Delta_{N}\right) \sqcup \Delta_{T}+(-1)^{\sum_{M \in V_{\Delta_{N}}} s_{M}} \Delta_{N} \sqcup\left(\partial \Delta_{T}\right) .
$$

Suppose first that $\Delta$ is in $\delta^{(p, 1)}$, then $\operatorname{Sym}\left(\partial \Delta_{T}\right)=0$. Indeed, if $p=0, \partial \Delta_{T}=0$, else $\partial \Delta_{T}$ contains two isolated arrows.

Now, if $\Delta$ is in $\delta^{(p, 0)}$, then $\partial \Delta_{T}=p \Delta_{T}^{\prime}$, where $\Delta_{T}^{\prime}$ contains one arrow and $p-1$ isolated points. Thus, after symmetrization,

$$
\begin{aligned}
0= & \partial \delta=\sum_{p \geqslant 0} \partial \delta^{(p, 0)}+\partial \delta^{(p, 1)} \\
= & \sum_{p \geqslant 0} \sum_{\Delta, p_{\Delta}=p, q_{\Delta}=0} c_{\Delta} \frac{1}{\left(v_{N}+1\right) ! p !} \operatorname{Sym}\left(\partial \Delta_{N} \sqcup \Delta_{T}+(-1)^{\sum s_{M}} p \Delta_{N} \sqcup \Delta_{T}^{\prime}\right) \\
& +\sum_{\Delta, p_{\Delta}=p, q_{\Delta}=1} \frac{1}{v_{N} !(p+1) !} c_{\Delta} \operatorname{Sym}\left(\partial \Delta_{N} \sqcup \Delta_{T}\right) .
\end{aligned}
$$

For any $\Delta$ in $\delta^{(p, 1)}$, define $g(\Delta)$ as the graph $\Delta_{N} \sqcup g\left(\Delta_{T}\right), g\left(\Delta_{T}\right)$ being the graph with $p+1$ isolated vertices and no edge (since there is no edge, the ordering of vertices is unessential). Then

$$
\partial\left(\frac{1}{p+1} g(\Delta)\right)=\frac{1}{p+1} \partial \Delta_{N} \sqcup g\left(\Delta_{T}\right)+(-1)^{\sum_{M \in V_{\Delta_{N}}} s_{M}} \Delta .
$$

Put

$$
\beta=\sum_{p \geqslant 0} \sum_{\Delta, p_{\Delta}=p, q_{\Delta}=1}(-1)^{\sum_{M \in V_{\Delta_{N}}} s_{M}} \frac{1}{v_{N} !(p+1) !} c_{\Delta} \operatorname{Sym}(g(\Delta)) .
$$

Then $(\delta-\partial \beta)^{(p, 1)}=0$ for any $p$. Put $\delta^{\prime}=\delta-\partial \beta=\sum c_{\Delta}^{\prime} \frac{1}{v_{N} ! p !} \operatorname{Sym}(\Delta)$. We then get 


$$
\begin{aligned}
0= & \partial \delta^{\prime}=\sum_{p \geqslant 0} \partial\left(\delta^{\prime}\right)^{(p, 0)} \\
= & \sum_{p \geqslant 0} \sum_{\Delta, p_{\Delta}=p} \frac{1}{\left(v_{N}+1\right) ! p !} c_{\Delta}^{\prime} \operatorname{Sym}\left(\partial \Delta_{N} \sqcup \Delta_{T}\right) \\
& +(-1)^{\sum_{M \in \Delta_{N}} s_{M}} \frac{p}{v_{N} !(p+1) !} c_{\Delta}^{\prime} \operatorname{Sym}\left(\Delta_{N} \sqcup \Delta_{T}^{\prime}\right) .
\end{aligned}
$$

Thus, for any $p>0$,

$$
0=\sum_{\Delta, p_{\Delta}=p}(-1)^{\sum_{M \in V_{\Delta_{N}}} s_{M}} \frac{p}{v_{N} !(p+1) !} c_{\Delta}^{\prime} \operatorname{Sym}\left(\Delta_{N} \sqcup \Delta_{T}^{\prime}\right) .
$$

Looking for ordering of edges for which the trivial arrow is at the end, we get, for any $p>0$,

$$
0=\sum_{\Delta, p_{\Delta}=p}(-1)^{\sum_{M \in V_{\Delta_{N}}} s_{M}} c_{\Delta}^{\prime} \operatorname{Sym}\left(\Delta_{N}\right) \sqcup \Delta_{T}^{\prime} .
$$

Therefore, $\sum_{\Delta, p_{\Delta}=p, \sum s_{M}=k} c_{\Delta}^{\prime} \operatorname{Sym}\left(\Delta_{N}\right) \sqcup \Delta_{T}=0$, and $\delta^{\prime}=\sum_{\Delta, p_{\Delta}=0} c_{\Delta}^{\prime} \Delta_{N}$ has no trivial component.

From now on, we suppose there is no trivial component in each $\Delta$.

For such a graph $\Delta$, it is natural to call hand a vertex $M$ such that $s_{M}=1, a_{M}=0$ and foot a vertex $M$ such that $s_{M}=0$ and $a_{M}=1$.

Definition 5.2 (The fh-degree of an aerial graph). The "feet and hands" degree (fh-degree) of $\Delta$, denoted fh $\operatorname{deg}(\Delta)$, is the number

$$
\text { fh } \operatorname{deg}(\Delta)=\#\left\{M \in V_{\Delta}, M \text { is a foot }\right\}-\#\left\{M \in V_{\Delta}, M \text { is a hand }\right\} .
$$

Let us remark that, for any graph, $\sum_{P \in V_{\Delta}} s_{P}-a_{P}=0$. Thus,

$$
0=\sum_{\substack{P \in V_{\Delta} \\ a_{P}+s_{P}=1}}\left(s_{P}-a_{P}\right)+\sum_{\substack{P \in V_{\Delta} \\ a_{P}+s_{P}>1}}\left(s_{P}-a_{P}\right),
$$

and

$$
\text { fh } \operatorname{deg}(\Delta)=\sum_{\substack{P \in V_{\Delta} \\ a_{P}+s_{P}=1}}\left(a_{P}-s_{P}\right)=\sum_{\substack{P \in V_{\Delta} \\ a_{P}+s_{P}>1}}\left(s_{P}-a_{P}\right) .
$$

If $\delta=\sum c_{\Delta, \mathfrak{n}}(\Delta, \mathfrak{n})$ is a linear combination of graphs, then we say that $\delta$ is homogeneous with fh-degree $b$ if fh $\operatorname{deg}(\Delta)=b$ for any $\Delta$ such that $c_{\Delta, \mathfrak{n}} \neq 0$.

Denote by $A G$ the space of linear combinations $\delta$, graded by $A G=\oplus_{b \in \mathbb{Z}} A G^{b}$, if $A G^{b}$ is the space of $\delta$ homogeneous with fh-degree $b$.

Suppose each $\Delta$ has no trivial component. Then for any $P$ in $V_{\Delta}$, the hands and feet of each graph in $\partial_{P}(\Delta, \mathfrak{n})$ are exactly the hands and feet of $\Delta$. Therefore,

Proposition 5.3 ( $\partial$ is homogeneous with fg-degree 0$)$. For any $b$, if $\delta$ belongs to $A G^{b}$, then $\partial \delta$ is in $A G^{b}$, or

$$
H^{*}(A G)=\bigoplus_{b \in \mathbb{Z}} H^{*}\left(A G^{b}\right)
$$

The first result of this paper will now be $H^{*}\left(A G^{b}\right)=0$, if $b \neq 0$. 


\section{Homotopy}

Let $(\Delta, \mathfrak{n})$ be a graph without any trivial component, a vertex $N$ in $V_{\Delta}$ such that $\left(a_{N}, s_{N}\right) \neq(1,1)$ or $\operatorname{Star}(N)=\operatorname{End}(N)=\{\overrightarrow{N N}\}$ is said to be non-simple. Thus, if $N$ is simple, then there are vertices $M$ and $R$ such that $N \neq M, N \neq R$ and $\operatorname{End}(N)=\{\overrightarrow{M N}\}, \operatorname{Star}(N)=\{\overrightarrow{N R}\}$.

Definition 6.1 (Homotopy). For any vertex $N$ if $N$ is not simple, define $h_{N}(\Delta, \mathfrak{n})$ $=0$.

If $N$ is simple, then

1. Put $N$ in the first place, multiplying by $\varepsilon_{N}(\mathfrak{n})=(-1)^{\sum_{M<N} s_{M}}$,

2. Suppress the vertex $N$ and the edge $\overrightarrow{N R}$,

3. Replace the arrow $\overrightarrow{M N}$ by $\overrightarrow{M R}$, keeping its position in $\operatorname{Star}(M)$.

The resulting oriented graph is

$$
h_{N}(\Delta, \mathfrak{n})=\varepsilon_{N}(\mathfrak{n})\left(h_{N}(\Delta), h_{N}(\mathfrak{n})\right) .
$$

The homotopy $h$ is the map defined by

$$
h(\Delta, \mathfrak{n})=\sum_{N \in V_{\Delta}} h_{N}(\Delta, \mathfrak{n}),
$$

which is linearly extended to linear combinations $\delta$ of graphs.

Example 6.2. Let us consider the following aerial graph:

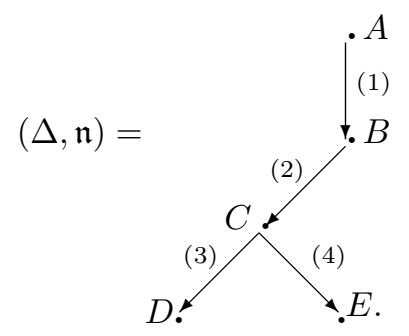

Then $h_{A}(\Delta, \mathfrak{n})=h_{C}(\Delta, \mathfrak{n})=h_{D}(\Delta, \mathfrak{n})=h_{E}(\Delta, \mathfrak{n})=0$ and

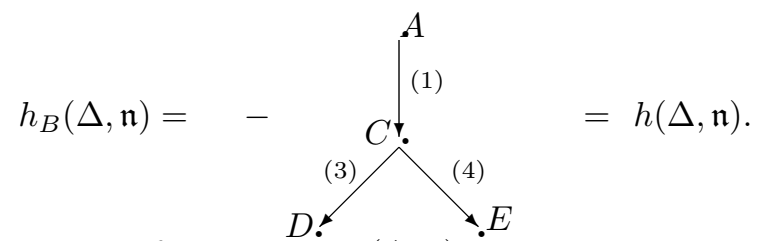

By construction, for any graph $(\Delta, \mathfrak{n})$ and any vertex $N$,

$$
\operatorname{Sym}\left(h_{N}(\Delta, \mathfrak{n})\right)=h_{N}(\operatorname{Sym}(\Delta, \mathfrak{n})) .
$$

Let us remark that the homotopy $h$ is homogeneous with fh-degree 0 :

$$
h\left(A G^{b}\right) \subset A G^{b} .
$$


Proposition 6.3 ( $h$ is a homotopy). For any homogeneous $\delta \in A G^{b}$, the following holds:

$$
(h \circ \partial+\partial \circ h)(\delta)=b \delta .
$$

Proof. Let us recall our notation:

(a) When computing $\partial_{P}(\Delta, \mathfrak{n})$, we add a new vertex, $Q: V_{\partial_{P} \Delta}=V_{\Delta} \sqcup\{Q\}$.

(b) When computing $h_{N}(\Delta, \mathfrak{n})$, the vertex $N$ disappears: $V_{h_{N}(\Delta)}=V_{\Delta} \backslash\{N\}$.

Thus,

$$
\begin{aligned}
(h \circ \partial+\partial \circ h)(\Delta, \mathfrak{n}) & \\
& =\sum_{\substack{N, P \in V_{\Delta} \\
N \neq P}}\left(h_{N} \circ \partial_{P}+\partial_{P} \circ h_{N}\right)(\Delta, \mathfrak{n})+\sum_{P \in V_{\Delta}}\left(h_{P} \circ \partial_{P}+h_{Q} \circ \partial_{P}\right)(\Delta, \mathfrak{n}) .
\end{aligned}
$$

If $N \neq P$ is not simple in $V_{\Delta}$, then $\left(h_{N} \circ \partial_{P}+\partial_{P} \circ h_{N}\right)(\Delta, \mathfrak{n})=0$.

If $N$ is simple, since $\delta$ is symmetric, then we can choose $\mathfrak{n}$ such that

$$
V_{\Delta}=\{N<P<A<\cdots<Z\} .
$$

Moreover, since $\Gamma=\left(h_{N} \circ \partial_{P}+\partial_{P} \circ h_{N}\right)(\Delta, \mathfrak{n})$ is symmetric under the position of $Q$, we only look for $Q$ such that $V_{\Gamma}=\{P<Q<A<\cdots<Z\}$.

In this case, there is no sign in $h_{N}(\Delta, \mathfrak{n})$ and, for any $t, I$ and $j$, the sign $\varepsilon_{P, Q, I, j}^{t}$ is $(-1)^{t-1}$. Moreover,

$$
\begin{aligned}
& \left(\partial_{P, Q, I, j} h_{N}(\Delta), \mathfrak{n}_{P, Q}^{t}\right) \\
& \quad=\left(\left(\vec{e}_{1}, \ldots, \vec{e}_{t-1}, \overrightarrow{P Q}_{1}, \ldots, \vec{e}_{j}\right),\left(\vec{e}_{j+1}^{\prime}, \ldots, \vec{e}_{s_{P}}^{\prime}\right), \operatorname{Star}^{\prime}(A), \ldots, \operatorname{Star}^{\prime}(Z)\right),
\end{aligned}
$$

where $\operatorname{Star}^{\prime}(M)$ is $\operatorname{Star}(M)$ except that the edge $\overrightarrow{M N}$ is replaced by $\overrightarrow{M R}$.

Let us remark that this computation holds even if $M=P$ or $R=P$.

Computing the corresponding term in $h_{N} \circ \partial_{P}$, we put $P$ in the first place, creating the sign $(-1)^{s_{P}}$, then proceed to the blow-up of $P$, creating the sign $\varepsilon_{P, Q, I, j}^{t}$, then put $P$ and $Q$ in their positions, passing through $N$, and finally, as above, the homotopy has no sign. We thus get the above signs multiplied by

$$
(-1)^{s_{P}}(-1)^{j+1}(-1)^{s_{P}-j}=-1 \text {. }
$$

Therefore,

$$
\sum_{\substack{N, P \in V_{\Delta} \\ N \neq P}}\left(h_{N} \circ \partial_{P}+\partial_{P} \circ h_{N}\right)(\Delta, \mathfrak{n})=0 .
$$

Let us compute $h_{P}\left(\partial_{P, Q, I, j} \Delta, \mathfrak{n}_{P, Q}^{t}\right)$. This term is not vanishing only if $P$ is simple, thus $j=0, t=1$ and $\# I=1$.

In this case, $\left(\partial_{P, Q,\{\vec{e}\}, 0} \Delta, \mathfrak{n}_{P, Q}^{1}\right)$ is a sum of graphs for each possible position of $Q$. By symmetry, it is enough to consider the graph where the vertices are

$$
\{P<Q<A<\cdots<Z\} \text {. }
$$

Then there is no sign in front of $h_{P}\left(\partial_{P, Q,\{\vec{e}\}, 0} \Delta, \mathfrak{n}_{P, Q}^{1}\right)=(\Delta, \mathfrak{n})$, with the change of labelling $P \mapsto Q$ for the first vertex. 
There is exactly $a_{P}$ such terms corresponding to the choice of the arrow $\vec{e}$ in $\operatorname{End}(P)$. Thus,

$$
\sum_{I} h_{P}\left(\partial_{P, Q, I=\{\vec{e}\}, 0} \Delta, \mathfrak{n}_{P, Q}^{1}\right)=a_{P}(\Delta, \mathfrak{n}) .
$$

Let us now compute $h_{Q}\left(\partial_{P, Q, I, j} \Delta, \mathfrak{n}_{P, Q}^{t}\right)$. This term is not vanishing only if $Q$ is simple, or $j=s_{P}-1$ and $I=\emptyset$. In this case, $1 \leqslant t \leqslant s_{P}$.

Remark that $\left(\partial_{P, Q, \emptyset, s_{P}-1} \Delta, \mathfrak{n}_{P, Q}^{t}\right)$ is a sum of graphs, with all possible positions for $Q$. By symmetry, we only consider the graph where the vertices are $\{P<Q<$ $A<\cdots<Z\}$. Then,

$$
h_{Q}\left(\partial_{P, Q, \emptyset, s_{P}-1} \Delta, \mathfrak{n}_{P, Q}^{t}\right)=(-1)^{t-1}(-1)^{s_{P}}\left(\Delta, \mathfrak{n}^{(t)}\right),
$$

where $\mathfrak{n}^{(t)}$ is obtained from $\mathfrak{n}$, just by pushing the $t^{\text {th }}$ arrow in $\operatorname{Star}(P)$ at the end of $\operatorname{Star}(P)$.

Replacing this arrow to its original place creates a sign $(-1)^{s_{P}-t}$. Thus, since there is $s_{P}$ such choices, we get

$$
\sum_{t} h_{Q}\left(\partial_{P, Q, \emptyset, s_{P}-1} \Delta, \mathfrak{n}_{P, Q}^{t}\right)=-s_{P}(\Delta, \mathfrak{n}) .
$$

This proves the proposition.

Corollary 6.4 (Restriction to fh-degree 0 graphs). Let $\delta$ be a symmetric combination of aerial graphs. If $\delta$ is a cocycle, then there is a coboundary $\partial \beta$ such that $\delta-\partial \beta$ is a cocycle homogeneous of fh-degree 0 :

$$
H^{*}(A G)=H^{*}\left(A G^{0}\right)
$$

There is a more precise localization of the cohomology given in the next section.

\section{Cutting the hands}

The arguments of this section were suggested by our referee.

Since the hands and feet of graphs in $\partial_{P}(\Delta, \mathfrak{n})$ are the same as those of $\Delta$ (with the same labelling), we now consider the subcomplex $\left(A G_{k}^{b}, \partial\right)$ of all combinations of graphs $\Delta$ with fh-degree $b$ and having $k$ hands. Let us now suppose that $k>0$.

Now, denote by $\widetilde{A G}_{k}^{b}$ the space of aerial graphs with fh-degree $b$ and having $k$ hands, with some extra labels $1, \ldots, N$ on the hand or foot vertices (it is clear that $N=b+2 k)$. To come back to $A G_{k}^{b}$, we have to forget this extra labelling, which means we restrict ourselves to $S_{N}$-invariant linear combinations of graphs in $\widetilde{A G}_{k}^{b}$, where $S_{N}$ is the symmetric group, acting by permuting the extra labels. Indeed, there is a complex isomorphism

$$
A G_{k}^{b} \cong\left(\widetilde{A G}_{k}^{b}\right)^{S_{N}}
$$

Moreover, for each subset $K$ in $\{1, \ldots, N\}$, with $\# K=k$, we put

$$
\widetilde{A G}_{K}^{b}=\operatorname{Span}\left\{\Delta \in \widetilde{A G}_{k}^{b} \text {, the extra labels on hands belong to } K\right\} \text {. }
$$

It is clear that, as complexes, $\widetilde{A G}_{k}^{b}=\bigoplus_{\# K=k} \widetilde{A G}_{K}^{b}$, and each subcomplex $\widetilde{A G}_{K}^{b}$ is isomorphic to $\widetilde{A G}_{[1, k]}^{b}([1, k]$ denotes the subset $\{1, \ldots, k\})$. 
Define now a mapping $\Phi$ from $\widetilde{A G}_{[1, k]}^{b}$ to $\widetilde{A G}_{[1, k-1]}^{b-2}$ by replacing the hand with extra label $k$ by a foot as follows:

Since we consider symmetric combination, we choose the labelling of vertices such that $A$ is the hand with extra label $k$ and $\operatorname{Star}(A)=\{\overrightarrow{A B}\}$. We define the mapping $\Phi$ as follows:

We change the arrow $\overrightarrow{A B}$ into $\overrightarrow{B A}$ and put this last arrow at the place $u$ in the new $\operatorname{Star}(B)$, multiplying the obtained graph, denoted $(\Phi(\Delta), \mathfrak{n}(u))$, by the sign $\eta^{u}$ of the vertices permutation placing $A, B$ in the two first places, and then replacing the vertices at their place. Explicitly,

$$
\eta^{u}= \begin{cases}(-1)^{u-1}(-1)^{\sum_{A<M<B} s_{M}} & \text { if } A<B, \\ (-1)^{u-1}(-1)^{s_{B}}(-1)^{\sum_{B<M<A} s_{M}} & \text { if } B<A .\end{cases}
$$

Finally, $\Phi(\Delta, \mathfrak{n})=\sum_{u=1}^{s_{B}+1} \eta^{u}(\Phi(\Delta), \mathfrak{n}(u))$. Remark that if $\delta$ is a symmetric linear combination of graphs, then $\Phi(\delta)$ is also symmetric.

Proposition 7.1 ( $\Phi$ is a complex morphism). $\Phi$ is a bijection from $\widetilde{A G}_{[1, k]}^{b}$ onto $\widetilde{A G}_{[1, k-1]}^{b-2}$. Moreover, $\Phi$ is an isomorphism of complexes. As a consequence,

$$
\widetilde{A G}_{k}^{b} \cong \widetilde{A G}_{0}^{-N} \otimes \mathbb{R}^{\left(\begin{array}{l}
N \\
k
\end{array}\right)} \text {. }
$$

Proof. First $\Phi$ is clearly a bijective map, and the inverse map $(\Delta, \mathfrak{n}) \mapsto \Phi^{-1}(\Delta, \mathfrak{n})$ is the following: In the graph $\Delta$, change the arrow $\overrightarrow{B A}$ into $\overrightarrow{A B}$. Then, in the ordering, place this last arrow at the place corresponding to the vertex $A$ and multiply by $\eta^{u}$ if $u$ is the place of $\overrightarrow{B A}$ in $\operatorname{Star}(B)$, in $\mathfrak{n}$.

Up to a symmetry on hands and feet, we can restrict ourselves to graphs $(\Delta, \mathfrak{n})$ where the ordering of vertices is $A<B<\cdots$. Let us compare $\Phi \circ \partial_{P}(\Delta, \mathfrak{n})$ and $\partial_{P} \circ$ $\Phi(\Delta, \mathfrak{n})$, if $P>B$.

Thanks to the symmetry property, we choose $Q$ just after $P$, let us fix $I \subset \operatorname{End}(P)$ and $j \leqslant s_{P}, 1 \leqslant t \leqslant j+1$; this choice defines a graph $\varepsilon_{P, Q, I, j}^{t}\left(\partial_{P, Q, I, j} \Delta, \mathfrak{n}_{P, Q}^{t}\right)$ in $\partial_{P}(\Delta, \mathfrak{n})$.

Applying $\Phi$ on this graph we get, for each $1 \leqslant u \leqslant s_{B}+1$, a graph

$$
\Gamma=\eta^{u} \varepsilon_{P, Q, I, j}^{t}\left(\Phi\left(\partial_{P, Q, I, j} \Delta\right), \mathfrak{n}_{P, Q}^{t}(u)\right) .
$$

With the same $Q, I, j, t$ and $u$, we have the graph

$$
\Gamma^{\prime}=\eta^{u} \varepsilon_{P, Q, I, j}^{t}\left(\partial_{P, Q, I, j}(\Phi(\Delta)), \mathfrak{n}(u)_{P, Q}^{t}\right) .
$$

Up to the sign, these two graphs coincide. But, by definition,

$$
\begin{aligned}
\varepsilon_{P, Q, I, j}^{t}=(-1)^{t-1}(-1)^{1+s_{B}+\sum_{B<M<P} s_{M}} & \\
& =(-1)^{t-1}(-1)^{\left(s_{B}+1\right)+\sum_{B<M<P} s_{M}}={\varepsilon^{\prime}}_{P, Q, I, j}^{t} .
\end{aligned}
$$

Consider now the case $P=B$. In any graph in $\Phi \circ \partial_{B}$ or $\partial_{B} \circ \Phi$, there is no edge $\overrightarrow{A B}$, but two new arrows $\overrightarrow{B Q}$ and either $\overrightarrow{B A}$ or $\overrightarrow{Q A}$.

Suppose there is $\overrightarrow{Q A}$ at the place $u$ in $\operatorname{Star}(Q)$ and $\overrightarrow{B Q}$ at the place $t$ in $\operatorname{Star}(B)$. The corresponding graph appears 
(a) in $\left(\Phi\left(\partial_{B, Q, I, j}(\Delta)\right), \mathfrak{n}^{t}(u)\right)$, only if $\overrightarrow{A B} \notin I$, with the sign

$$
(-1)^{j+1} \eta^{u} \varepsilon_{B, Q, I, j}^{t}=(-1)^{j+1}(-1)^{u-1}(-1)^{t-1}(-1)^{s_{A}}=(-1)^{t+u+j},
$$

(b) in $\left(\partial_{B, Q, I, j}(\Phi(\Delta)), \mathfrak{n}(j+u)^{t}\right)$ with the sign

$$
\eta^{j+u-1} \varepsilon_{B, Q, I, j}^{t}=(-1)^{j+u-1}(-1)^{t-1}=(-1)^{j+u+t} .
$$

Similarly, suppose there is $\overrightarrow{B A}$ at the place $u$ in $\operatorname{Star}(B)$ and $\overrightarrow{B Q}$ at the place $t$ in $\operatorname{Star}(B)$.

1. The corresponding graph appears in $\left(\Phi\left(\partial_{B, Q, I, j}(\Delta)\right), \mathfrak{n}^{t^{\prime}}(u)\right)$, only if $\overrightarrow{A B} \in I$,

(a) if $t<u$, with $t^{\prime}=t$ and the sign

$$
\eta^{u} \varepsilon_{B, Q, I, j}^{t}=(-1)^{u-1}(-1)^{t-1}(-1)^{s_{A}}=(-1)^{t+u+1},
$$

(b) if $t>u$ with $t^{\prime}=t-1$, and the sign

$$
\eta^{u} \varepsilon_{B, Q, I, j}^{t-1}=(-1)^{u-1}(-1)^{t-2}(-1)^{s_{A}}=(-1)^{t+u} .
$$

2. The corresponding graph also appears

(a) if $t<u$, in $\left(\partial_{B, Q, I \backslash\{\overrightarrow{A B}\}, j+1}(\Phi(\Delta)), \mathfrak{n}(u-1)^{t}\right)$, with the sign

$$
\eta^{u-1} \varepsilon_{B, Q, I \backslash\{\overrightarrow{A B}\}, j+1}^{t}=(-1)^{u-2}(-1)^{t-1}=(-1)^{u+t+1},
$$

(b) if $t>u$, in $\left(\partial_{B, Q, I \backslash\{\overrightarrow{A B}\}, j+1}(\Phi(\Delta)), \mathfrak{n}(u)^{t}\right)$, with the sign

$$
\eta^{u} \varepsilon_{B, Q, I \backslash\{\overrightarrow{A B}\}, j+1}^{t}=(-1)^{u-1}(-1)^{t-1}=(-1)^{u+t} .
$$

This finally proves

$$
\Phi \circ \partial=\partial \circ \Phi .
$$

By iteration of this construction, we get the following isomorphisms for each subset $K$ in $\{1, \ldots, N\}$, with $\# K=k$ :

$$
\widetilde{A G}_{K}^{b} \cong \widetilde{A G}_{[1, k]}^{b} \cong \widetilde{A G}_{\emptyset}^{-N}=\widetilde{A G}_{0}^{-N} .
$$

This proves the proposition.

The homotopy is defined on $\widetilde{A G}_{0}^{-N}$ as well. Therefore, if $N>0$, then $\widetilde{A G}_{0}^{-N}$ is acyclic, thus $\widetilde{A G}_{k}^{b}$ is acyclic.

Since taking invariants of a finite group action commutes with taking cohomology, we get, if $N>0$,

$$
H^{*}\left(A G_{k}^{b}\right)=H^{*}\left(\left(\widetilde{A G}_{k}^{b}\right)^{S_{N}}\right)=H^{*}\left(\left(\widetilde{A G}_{k}^{b}\right)\right)^{S_{N}}=0 .
$$

Summarizing, we finally get the following localization of the Chevalley cohomology $H^{*}(A G)$.

Theorem 7.2 (Localization of the cohomology). Let $A G_{k}^{b}$ be the space of graphs with fh-degree $b$ and $k$ hands. Then for any $b \neq 0$ or $k>0, H^{*}\left(A G_{k}^{b}\right)=0$. Thus the cohomology is localized into the space of graphs without trivial component and without any foot or hand:

$$
H^{*}(A G)=H^{*}\left(A G_{0}^{0}\right)
$$




\section{Application to some quotient complexes}

In this section, we consider the space $T_{\text {poly }}^{\text {hom }}\left(\mathbb{R}^{d}\right)$ of strongly homogeneous polyvector fields $\alpha$, i.e., polyvector fields:

$$
\alpha=\sum_{1 \leqslant i_{1}, \ldots, i_{k} \leqslant d} \alpha^{i_{1} \cdots i_{k}}(x) \partial_{x_{i_{1}}} \wedge \cdots \wedge \partial_{x_{i_{k}}}
$$

where $\alpha^{i_{1} \cdots i_{k}}(x)$ is a homogeneous polynomial with degree $k$.

This space is a subalgebra of $\left(T_{\text {poly }}\left(\mathbb{R}^{d}\right),[,]_{S}\right)$. Let us look to its cohomology with value in the trivial module.

Let us say that a graph $\Delta$ is strongly homogeneous if $s_{M}=a_{M}$ for any vertex $M$.

Denote by $H G$ the space of symmetric linear combination of strongly homogeneous graphs and by $N H G$ the space of symmetric linear combination of non-homogeneous graphs.

In fact, the space $H G$ naturally appears in the computation of the cohomology: the linear combinations $\delta$ of strongly homogeneous aerial graphs define cochains $C_{\delta}$ on $T_{\text {poly }}^{\text {hom }}\left(\mathbb{R}^{d}\right)$ with value in the base field $\mathbb{R}$.

Lemma 8.1 ( $H G$ is a quotient complex with a homotopy). The space $N H G$ is a subcomplex of $A G$, and $H G \cong A G / N H G$ is a quotient complex. More precisely,

$$
\partial(N H G) \subset N H G, \quad \text { and } \quad h(N H G) \subset N H G .
$$

Proof. Suppose $\Delta$ is non-homogeneous, $s_{P} \neq a_{P}$ for some vertex $P$ in $\Delta$; then the only point to verify is the existence of a non-homogeneous vertex in $\partial_{P} \Delta=$ $\sum \pm \partial_{P, Q, I, j} \Delta$. But in this last graph, either $P$ or $Q$ is non-homogeneous.

By definition of the homotopy, $h=\sum h_{N}$, we only look to simple vertices $N$ in $\Delta$, clearly the non-homogeneous vertex $P$ in $\Delta$ remains non-homogeneous in $h_{N}(\Delta)$.

Let $H_{\text {hom }}^{*}(H G)$ denote the cohomology of the quotient complex. For instance, each odd wheel is a cocycle.

Definition 8.2 (Wheels). A simple wheel is a graph $\Delta_{p}$ with $p$ vertices $A_{1}, \ldots, A_{p}$ and $p$ edges $\overrightarrow{A_{1} A_{2}}, \ldots, \overrightarrow{A_{p-1} A_{p}}$, and $\overrightarrow{A_{p} A_{1}}$. Denote its symmetrization by $W_{p}=$ $\operatorname{Sym}\left(\Delta_{p}\right)$.

We can represent $\Delta_{p}$ by the following:

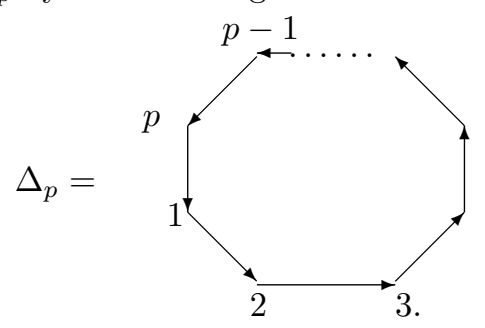

Remark 8.3. The symmetric even wheels vanish: $W_{2 p}=0$.

An easy computation proves that each odd wheel $W_{2 p+1}$ is a cocycle in $H G$. For instance, 
Example 8.4.

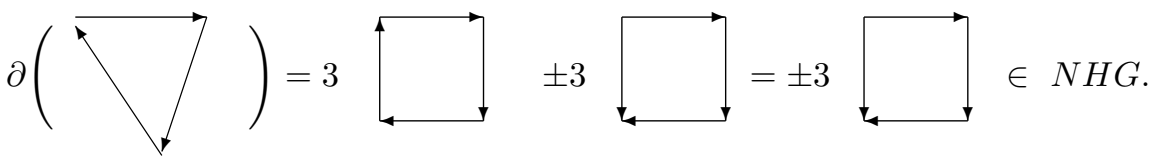

Now, if $W_{2 p+1}$ is a coboundary in $H G$, it would be the coboundary of a strongly homogeneous connected graph with $2 p$ edges and $2 p$ vertices, that is of the wheel $W_{2 p}$, but this wheel vanishes. Therefore,

$$
H_{\text {hom }}^{*}(H G) \neq H^{*}\left(A G_{0}^{0}\right)=H^{*}(A G)
$$

since, for instance, $H^{3}(A G)=0$ (see $[\mathbf{K} 1, \mathbf{A G M}]$ ).

Recall that the odd wheels freely generate the cohomology of linear and vectorial graphs; see [AAC1, AAC2]. In order to generalize the linear case, we now consider a new quotient complex of graphs, the space of ascending graphs.

A graph $\Delta$ is ascending if, for each vertex $M$, with $s_{M}$ arrows coming out from $M$ and $a_{M}$ arrows coming in to $M$, we have

$$
\begin{cases}\text { either } & \left(a_{M}, s_{M}\right) \in\{(0,0),(1,0),(0,1),(1,1)\}, \\ \text { or } & \left(a_{M}, s_{M}\right) \in\{(a, s), \quad \text { such that } a<s, \text { and } 1<s\} .\end{cases}
$$

In the first case, we shall say that $M$ is type 1 , and in the second case, we say $M$ is type 2 .

Let us denote the space of these graphs by $A S C$, and the space of non-ascending graphs by $N A S C$.

Example 8.5. The following graph is an ascending graph:

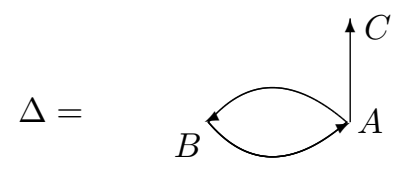

because $\left(a_{A}, s_{A}\right)=(1,2),\left(a_{B}, s_{B}\right)=(1,1)$ and $\left(a_{C}, s_{C}\right)=(1,0)$.

Conversely, the following graph is non-ascending:

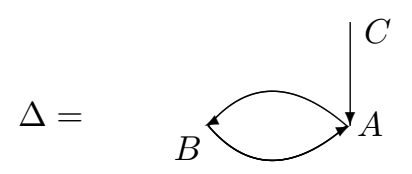

since $\left(a_{A}, s_{A}\right)=(2,1)$, and the vertex $A$ is neither type 1 nor type 2 .

Lemma 8.6 ( $A S C$ is a quotient complex with a homotopy). The space $N A S C$ is a subcomplex of $A G$, and $A S C \cong A G / N A S C$ is a quotient complex. More precisely,

$$
\partial(N A S C) \subset N A S C \quad \text { and } \quad h(N A S C) \subset N A S C .
$$


Proof. Let $\Delta$ be a non-ascending graph; there is a vertex $P$ in $\Delta$ that does not satisfy the conditions of the definition. This means $a_{P} \geqslant s_{P}$ and $a_{p} \geqslant 2$.

If $M \neq P$, then each graph in $\partial_{M} \Delta$ is still non-ascending in $P$. In $\partial_{P} \Delta$, the quantity $\left(a_{M}, s_{M}\right)$ changes only for $M=P$ or $M=Q$. Denote by $s_{P}^{\prime}, s_{Q}^{\prime}$ (resp. $\left.a_{P}^{\prime}, a_{Q}^{\prime}\right)$ the number of edges starting from $P, Q$ (resp. incoming in $P, Q$ ) for a graph in $\partial_{P} \Delta$. We have:

$$
a_{P}^{\prime}+a_{Q}^{\prime}=a_{P}+1, \quad s_{P}^{\prime}+s_{Q}^{\prime}=s_{P}+1, \quad a_{P}^{\prime}+s_{P}^{\prime} \geqslant 2, \quad a_{Q}^{\prime}+s_{Q}^{\prime} \geqslant 2 .
$$

Suppose that the vertex $P$ is ascending. Then

(a) either $\left(a_{P}^{\prime}, s_{P}^{\prime}\right)=(1,1)$ thus $\left(a_{Q}^{\prime}, s_{Q}^{\prime}\right)=\left(a_{P}, s_{P}\right)$,

(b) or $a_{P}^{\prime}<s_{P}^{\prime}$ thus $a_{Q}^{\prime}-s_{Q}^{\prime}>a_{P}-s_{P} \geqslant 0$.

In both cases, due to its new vertex $Q, \partial_{P} \Delta$ is non-ascending.

By definition of the homotopy, $h=\sum h_{N}$, where the sum is on the simple vertices $N$ in $\Delta$, clearly, if $\Delta$ is non-ascending with its vertex $P$, then $h_{N}(\Delta)$ remains a non-ascending due to the same vertex $P$.

Definition 8.7 (Lines). A simple line $\Delta_{r}$ is a graph with $r+1$ vertices $A_{1}, \ldots, A_{r+1}$ and $r$ edges $\overrightarrow{A_{r+1} A_{r}}, \overrightarrow{A_{r} A_{r-1}}, \ldots, \overrightarrow{A_{2} A_{1}}$. We denote by $L_{r}$ the symmetrization of $\Delta_{r}$.

Let us now consider a connected ascending graph $\Delta$ with only type 1 vertices. Then $\Delta$ is either a wheel or a line. These graphs and their cohomology were studied, for instance, in [AAC1].

The ascending graphs complex has the same cohomology as the complex of linear or vectorial graphs:

Corollary 8.8 (Cohomology of ascending graphs). The cohomology of the quotient complex $H^{*}(A S C)$ is freely generated by the products of odd wheels:

$$
H^{*}(A S C)=\left\langle W_{2 k_{1}+1} \wedge W_{2 k_{2}+1} \wedge \cdots \wedge W_{2 k_{p}+1}, 0<k_{1}<\cdots<k_{p}\right\rangle .
$$

Proof. Denote by $k$ the number of hands and by $\ell$ the number of feet of the graphs in $A S C$. Let $A S C_{k, \ell}$ the vector space generated by such graphs. Since, for each graph, $\sum_{P} a_{P}=\sum_{P} s_{P}$, we can write

$$
A S C=A S C^{\text {Type } 1} \oplus \sum_{k<\ell} A S C_{k, \ell}
$$

where $A S C^{\text {Type } 1}$ is the space generated by graphs having only type 1 vertices, that is, the product of lines and wheels.

Since the homotopy is defined on the quotient, we get $H^{*}\left(A S C_{k, \ell}\right)=0$ if $k<\ell$ and $H^{*}(A S C)=H^{*}\left(A S C^{\text {Type } 1}\right)$.

In $[\mathbf{A A C 1}]$, it is proved that the cohomology of lines vanishes and the cohomology of wheels is the space generated by the products $W_{2 k_{1}+1} \wedge W_{2 k_{2}+1} \wedge \cdots \wedge W_{2 k_{p}+1}$, with $0<k_{1}<\cdots<k_{p}$. 
Remark 8.9. Similarly, we can study the space of descending graphs where the vertices $M$ are type $\left(a_{M}, s_{M}\right)$, with

$$
\begin{cases}a_{M}>s_{M} & \text { if } a_{M}>1 \\ s_{M} \leqslant 1 & \text { if } a_{M} \leqslant 1\end{cases}
$$

As above, the cohomology is freely generated by the product of odd wheels $W_{2 p+1}$.

\section{References}

[AAC1] W. Aloulou, D. Arnal and R. Chatbouri, Cohomologie de Chevalley des graphes vectoriels, Pacific J. Math. 229 (2007), no. 2, 257-292.

[AAC2] W. Aloulou, D. Arnal and R. Chatbouri, Chevalley cohomology for linear graphs, Lett. Math. Phys. 80 (2007), no. 2, 139-154.

[AGM] D. Arnal, A. Gammella and M. Masmoudi, Chevalley cohomology for Kontsevich's graphs, Pacific J. Math. 218 (2005), no. 2, 201-239.

[K1] M. Kontsevich, Formal (non)-commutative symplectic geometry, in The Gelfand Mathematical Seminars 1990-1992 (L. Corwin, I. Gelfand, and J. Lepowsky, eds.), 173-187, Birkhäuser Boston, Boston, MA, 1993.

[K2] M. Kontsevich, Deformation quantization of Poisson manifolds, Lett. Math. Phys. 66 (2003), no. 3, 157-216.

Walid Aloulou Walid.Aloulou@ipeim.rnu.tn

Université de Sousse, Laboratoire de Mathématique Physique Fonctions Spéciales et Applications, and Département de Mathématiques, Institut Préparatoire aux Etudes d'Ingénieurs de Sfax, Route Menzel Chaker Km 0.5, Sfax, 3018, Tunisie

Didier Arnal Didier.Arnal@u-bourgogne.fr

Institut de Mathématiques de Bourgogne, UMR CNRS 5584, Université de Bourgogne, U.F.R. Sciences et Techniques B.P. 47870, F-21078 Dijon Cedex, France

Ridha Chatbouri Ridha.Chatbouri@ipeim.rnu.tn

Université de Sousse, Laboratoire de Mathématique Physique Fonctions Spéciales et Applications and Département de Mathématiques, Faculté des Sciences de Monastir, Avenue de l'environnement, 5019 Monastir, Tunisie 